\title{
Clarifying the new problem for quantum mechanics: Reply to Vaidman
}

\author{
Alexander Meehan
}

\begin{abstract}
I respond to Vaidman's recent criticisms of my paper “A New Problem for Quantum Mechanics".
\end{abstract}

\section{Introduction}

In a recent letter, Lev Vaidman [1] raises some objections to my forthcoming article [2], in which I argue that there is a new problem for quantum mechanics which is distinct from the standard measurement problem. I am grateful for Vaidman's criticisms, as they provide the opportunity to clarify a key aspect of my argument. Indeed, Vaidman's two main objections are based on a misreading of my thesis which I hope to correct here. I also briefly address Vaidman's remaining, more minor, objections.

\section{The new problem involves measurement, too}

The purpose of my [2] was to introduce and argue for a new problem, the control problem, defined as the incompatibility of the following four claims:

(B1) Preparation: We can successfully prepare quantum states: at least some of our preparation devices are such that, if determinately fed many inputs, they output a non-trivial 
fraction of those inputs in some specified range of quantum states. (Here the 'inputs' are subsystems, and we define 'the quantum state of a subsystem' in the standard way, as its reduced state.)

(B2) Unitarity: The quantum state of an isolated system always evolves in accord with a deterministic dynamical equation that preserves the inner product, such as the Schrödinger equation.

(B3) Determinate Input: It is always determinate whether or not a subsystem has been input into a given (measuring or preparation) device.

(B4) Competent Measurement: There is always, from a first-person view, an experienced 'observed outcome' of a measurement, even if-from a 'God's eye view' - there is no unique determinate outcome. Moreover, our measuring devices are at least somewhat informative and reliable. For example, if a spin- $z$ measuring device is determinately fed an electron in the eigenstate $\uparrow_{z}$, it will output 'UP' (and not 'DOWN').

The reader may notice that this fourth assumption, (B4), is about measurement. Yet, the control problem is supposed to be "a new problem" which is "distinct from the standard measurement problem". How can it be "new" if it still involves measurement?

It is new because it does not rely on the following assumption:

(A1) Completeness: The quantum state of a system (e.g. of a measuring device) determines, directly or indirectly, all of its physical properties.

The standard measurement problem, and its usual variants, crucially rely on this no-hiddenvariables-type assumption [3]. The novelty of the control problem is that it relies on (B1) rather than (A1): a tension with unitarity and determinateness exists even if the wave function does not specify everything, as long as Preparation is assumed. Roughly, this is because we can associate each measurement outcome with a distinct preparation procedure, and then the prepared quantum states can be used as a proxy for the pointer eigenstates. 
This tension is worth noting because we might have a candidate theory which rejects (A1) and thus avoids the inconsistency of the standard measurement problem, but still falls prey to the inconsistency (B1)-(B4).

Though the control problem is new in this sense, it still involves measurement. Thus I emphasize, though perhaps not sufficiently strongly [2, Sect. 1]:

"On the other hand, the control problem invokes Competent Measurement, and crucially involves a measurement-type interaction (combined with a preparation). So if the measurement problem is defined as the general puzzle that measurement interactions can be problematic under Schrodinger dynamics, then the control problem should be viewed as a specific aspect of it."

\section{Vaidman's reading}

Vaidman [1] attributes to my article a much stronger and less plausible thesis: that there is an internal inconsistency between preparation and unitarity, and in particular that (B1)(B3) are incompatible on their own, without any consideration of measurement. Indeed, Vaidman omits (B4) in his exposition. When the role of measurement in my problem later arises, he remarks that "when introducing a new problem in quantum mechanics he was not supposed to include measurement, which is problematic by itself". Of course, my goal was not to set measurement aside, but to point out that a problem can arise even without (A1) Completeness. This key point about Completeness is not mentioned anywhere by Vaidman.

With this clarification in mind, we can straightforwardly address Vaidman's main criticisms:

“ There exists a particular set-up in which all Meehan's claims are found to be true, so we prove by construction that his inconsistency proof fails.

- Demonstrating his 'new problem' Meehan presents a set-up involving measurement (which is known to be a problem in quantum mechanics) together 
with preparation. He tries to derive the inconsistency from the preparation of states, but makes an error (dividing by zero) in his proof."

Let us take these two points in turn.

\subsection{Unitary preparation and measurement}

Vaidman's alleged counterexample involves a set-up in Bohmian mechanics, where a pure spin state is successfully prepared through a unitary operation. There is no measurement in his set-up.

I agree that there is no inconsistency without (B4). In fact, I give a similar example of a unitary preparation in Sect. 3.1 of my paper. As I write there, "unitarity and the preparation assumption are perfectly compatible. Schrodinger's theory does not face the same general problem for preparations as it does for measurements" (see also [4]).

The set-up for my problem is a measurement followed by a preparation, where which state is prepared depends on the observed outcome. On a Bohmian analysis, it is indeed true that the systems are not prepared in the desired reduced state, but rather in a desired conditional or effective state $[5,6]$. So in my terminology, Bohmian mechanics rejects (B1) in favor of:

(B1') Conditional Preparation: Given determinate inputs, our preparation devices can prepare those inputs in desired conditional quantum states (relativized to an extra parameter) but not desired quantum states simpliciter.

Here the 'extra parameter' involves the final Bohmian macro-configuration of the pointer, with which the choice of preparation was correlated. As I discuss in [2], I think this solution to the control problem is one of the most elegant.

\subsection{Measurement, orthogonality, and "dividing by zero"}

In analyzing my incompatibility argument in greater detail, Vaidman writes: 
"The inner product of the prepared state is not relevant for calculating the inner product between the final states of the whole composite system for the two alternatives: it is zero due to the measurement which is embedded in the set-up."

Here Vaidman is saying that measurement is already incompatible with the preservation of inner products, (B2) Unitarity, assuming determinateness, and so the role of preparation in my proof is redundant. I suspect that Vaidman is implicitly relying on (A1) Completeness to get this conclusion. Indeed, he seems to be assuming that since the two alternatives involve distinct macroscopic measurement outcomes, they must be represented by orthogonal wave functions. But this is precisely the kind of assumption that a theorist who rejects (A1) might deny. For example, in a Bohmian model of the spin- $z$ measurement of an $\uparrow_{x}$ particle, the two alternatives of an 'UP' and 'DOWN' outcome have the same final wave function. (It's only if we condition this wave function on the final macro-configuration of the device (the Bohmian positions) that we get orthogonality.)

Of course, I agree that once Completeness is assumed, we will run into tensions with unitarity for completely familiar reasons. The point is to demonstrate a tension that does not hinge on Completeness, and for this, my preparation assumption plays a crucial role and is not redundant.

Vaidman also claims that I make a "technical error" in my proof: "[he] cannot claim that Eq. 8 implies Eq. 9". My reasoning was:

"Unitarity implies [toward contradiction],

$$
\left|\left(\uparrow_{z}, \uparrow_{x}\right)\right|^{2} \cdot \mid\left.(\text { initial, initial })\right|^{2}=\mid\left.(\text { final, final' })\right|^{2} \cdot\left|\left(\uparrow_{z}, \uparrow_{x}\right)\right|^{2 \cdot M} .
$$

which, since $\mid\left.($ initial, initial $)\right|^{2}=1$ and $\mid\left.($ final, final' $)\right|^{2} \leq 1$, implies:

$$
\left|\left(\uparrow_{z}, \uparrow_{x}\right)\right|^{2} \leq\left|\left(\uparrow_{z}, \uparrow_{x}\right)\right|^{2 \cdot M} . "
$$


Vaidman's objection is that $\mid\left.($ final, final' $)\right|^{2}=0$ and so in concluding Eq. 9 I am "dividing by zero". But there is no division going on. Consider the LHS and RHS of Eq. 8. By $\mid\left.($ initial, initial $)\right|^{2}=1$, the LHS equals $\left|\left(\uparrow_{z}, \uparrow_{x}\right)\right|^{2}$. By $\mid\left.($ final, final' $)\right|^{2} \leq 1$, the RHS is less than or equal to $\left|\left(\uparrow_{z}, \uparrow_{x}\right)\right|^{2 \cdot M}$. So if the LHS and RHS are equal, it follows that $\left|\left(\uparrow_{z}, \uparrow_{x}\right)\right|^{2} \leq$ $\left|\left(\uparrow_{z}, \uparrow_{x}\right)\right|^{2 \cdot M}$ as claimed.

\section{Remaining objections}

Finally, Vaidman advances two additional criticisms, which I briefly address:

" - There is no compelling reason to accept one of the statements [in the alleged inconsistency], so the inconsistency, if it exists, does not represent a problem for quantum mechanics. [...]

- Finally, in his critical comment regarding the no-cloning theorem of quantum mechanics, he confuses approximate cloning with precise cloning."

\subsection{The determinateness assumption}

Vaidman claims that the statement (B3), Determinate Input, is implausible because it concerns a quantum microscopic subsystem which should not be expected to have determinate classical features. For convenience, here is the assumption once more:

(B3) Determinate Input: It is always determinate whether or not a subsystem has been input into a given (measuring or preparation) device.

I agree with Vaidman that the "always" here is probably too strong. But at least in the context of my set-up, the assumption is not implausible. In my set-up, there are two preparation devices, one (call it $D$ ) designed to prepare $D$-states, the other (call it $D^{\prime}$ ) designed to prepare $D^{\prime}$-states. An experimenter measures the spin- $z$ of an electron. Her protocol is that if she obtains 'UP', she feeds an ensemble of systems into $D$; otherwise, she 
feeds them into $D^{\prime}$. Suppose the lab is arranged so that $D$ and $D^{\prime}$ are on opposite (left and right) sides of the room. (B3) then says that there is a determinate fact as to whether the experimenter goes to the left side and uses $D$, or goes to the right side and uses $D^{\prime}$. Of course, some interpretations, such as Everettian mechanics, will ultimately want to reject this assumption (on this interpretation, on one branch she uses $D$ while on another she uses $\left.D^{\prime}\right)$. But it is at least initially plausible.

\subsection{State determination and approximate versus precise cloning}

In my paper, I also analyze a common argument against the possibility of individual state determination given by Vaidman and others [7, 8]: if we could reliably determine the previously unknown state of a system given only a single copy, then we could prepare many more copies, thus violating the no-cloning theorem. I point out that this argument generalizes to any finite number of copies: if we could reliably determine the previously unknown state given $N$ copies (for arbitrary finite $\mathrm{N}$ ), then we could prepare many more $M \gg N$ copies, thus violating the no-cloning theorem. I then remark:

"Unless 'determining' is read in an overly stringent way, this result is absurd. When $N$ is large, we can reliably determine the initial wave function. So something has gone wrong [with the argument]."

I suggest that what has gone wrong is that the reasoning of $[7,8]$ is implicitly taking (B1)(B3) for granted. Since these assumptions conflict with (B4), it is unsurprising we are able to get this absurd result to follow.

In response, Vaidman writes:

"Although Meehan considers this statement as 'absurd', it is correct. We cannot clone an unknown quantum state even if have a finite number $N$ of systems with this state. [...] Exact tomography of a quantum state requires unlimited number of copies. A finite ensemble allows only for approximate determination." 
Vaidman is clearly adopting what I called an "overly stringent" reading of 'determine'. Of course, I agree that on this stringent reading, the statement is not absurd. My point is that (B1)-(B3), which are presumed in the argument, conflict even with approximate determination (see [2, Sect. 6.1], especially the discussion of (B4') Competent State Determination).

Let me make one further clarificatory remark about approximate versus exact cloning. It is well known [9] that there is a nice connection between the fidelity bounds on state estimation and the bounds on imperfect cloning. These bounds depend in the expected way on $N$; there are indeed strong cloning-based considerations against individual state determination, and more generally against reliable estimation for low $N$. Crucially, however, this type of cloning produces a high degree of entanglement/correlation between the outputs [9], and so we should not confuse it with the type of cloning (resulting from the successful preparation of an ensemble) discussed above.

\section{Conclusion}

Vaidman ends his remarks by noting "The measurement problem of quantum theory is still with us. It has many (sometimes contradicting) solutions in various interpretations with, unfortunately, no consensus yet about the preferred one." On this we are in agreement. The measurement problem remains as pressing as ever. Whether one views Completeness as a defining feature of this problem will affect whether one views the control problem as separate, or yet another species of it. But in either case, I take this additional problem, which involves both measurement and preparation, to be worth pointing out.

\section{References}

[1] Lev Vaidman. There is no new problem for quantum mechanics. Foundations of Physics, forthcoming. Published online Oct 2020. DOI: 10.1007/s10701-020-00394-w. 
[2] Alexander Meehan. A new problem for quantum mechanics. The British Journal for the Philosophy of Science, forthcoming. Published online Dec 2019. DOI: 10.1093/bjps/axz053.

[3] Tim Maudlin. Three measurement problems. Topoi, 14(1):7-15, 1995.

[4] Linda Wessels. The preparation problem in quantum mechanics. In John Earman and John Norton, editors, The Cosmos of Science, pages 243-273. University of Pittsburgh Press, 1997.

[5] Detlef Dürr, Sheldon Goldstein, and Nino Zanghí. Quantum equilibrium and the origin of absolute uncertainty. Journal of Statistical Physics, 67(5-6):843-907, 1992.

[6] Travis Norsen. Bohmian conditional wave functions (and the status of the quantum state). In Journal of Physics: Conference Series, volume 701, page 012003. IOP Publishing, 2016.

[7] G. M. D'Ariano and H. P. Yuen. Impossibility of measuring the wave function of a single quantum system. Phys. Rev. Lett., 76:2832-2835, 1996.

[8] Lev Vaidman. Protective measurement of the wave function of a single system. In Shan Gao, editor, Protective Measurement and Quantum Reality, pages 15-26. Cambridge University Press, 2015.

[9] Joonwoo Bae and Antonio Acín. Asymptotic quantum cloning is state estimation. Physical Review Letters, 97(3):030402, 2006. 